\title{
Canonical Graph Contractions of Linear Relations on Hilbert Spaces
}

\author{
Zsigmond Tarcsay $^{1,2}\left(D \cdot\right.$ Zoltán Sebestyén $^{1}$
}

Received: 1 July 2020 / Accepted: 30 November 2020 / Published online: 20 January 2021

(C) The Author(s) 2021

\begin{abstract}
Given a closed linear relation $T$ between two Hilbert spaces $\mathcal{H}$ and $\mathcal{K}$, the corresponding first and second coordinate projections $P_{T}$ and $Q_{T}$ are both linear contractions from $T$ to $\mathcal{H}$, and to $\mathcal{K}$, respectively. In this paper we investigate the features of these graph contractions. We show among other things that $P_{T} P_{T}^{*}=\left(I+T^{*} T\right)^{-1}$, and that $Q_{T} Q_{T}^{*}=I-\left(I+T T^{*}\right)^{-1}$. The ranges $\operatorname{ran} P_{T}^{*}$ and ran $Q_{T}^{*}$ are proved to be closely related to the so called 'regular part' of $T$. The connection of the graph projections to Stone's decomposition of a closed linear relation is also discussed.
\end{abstract}

Keywords Linear relation - Unbounded operator · Multivalued operator · Closed operator · Graph contraction · Stone decomposition

Mathematics Subject Classification Primary 47A05

Dedicated to Henk de Snoo on the occasion of his 75th birthday.

Communicated by Seppo Hassi.

The corresponding author Zs. Tarcsay was supported by DAAD-TEMPUS Cooperation Project "Harmonic Analysis and Extremal Problems" (Grant No. 308015), by the János Bolyai Research Scholarship of the Hungarian Academy of Sciences, and by the ÚNKP-20-5-ELTE-185 New National Excellence Program of the Ministry for Innovation and Technology. "Application Domain Specific Highly Reliable IT Solutions" project has been implemented with the support provided from the National Research, Development and Innovation Fund of Hungary, financed under the Thematic Excellence Programme TKP2020-NKA-06 (National Challenges Subprogramme) funding scheme.

This article is part of the topical collection "Recent Developments in Operator Theory- Contributions in Honor of H.S.V. de Snoo” edited by Jussi Behrndt and Seppo Hassi.

\section{Zsigmond Tarcsay}

tarcsay@cs.elte.hu

Extended author information available on the last page of the article 


\section{Introduction}

When dealing with (unbounded) operators, it is sometimes beneficial to identify them with their graph, that is, to treat them as linear subspaces of the corresponding product space. This approach is especially useful if the operator in question is non-closable, that is, when the closure of its graph is not the graph of a 'single-valued' operator anymore. In addition, the adjoint of a linear transformation can be interpreted as an operator only if it is densely defined.

The theory of linear relations (or 'multi-valued' linear operators in other words) between Hilbert spaces goes back at least to the fundamental paper by Arens [1]. By definition, a linear relation $T$ between two Hilbert spaces $\mathcal{H}$ and $\mathcal{K}$ is just a vector subspace of the product Hilbert space $\mathcal{H} \times \mathcal{K}$. In this way, the only (but significant) difference between operators and relations is that $\{0, k\} \in T$ does not necessarily imply $k=0$. However, this generality greatly simplifies the handling of operations such as taking closure, adjoint, or inverse.

A linear relation $T$ consists of certain ordered pairs $\{x, y\}$ of $\mathcal{H} \times \mathcal{K}$, so one may consider the first and second coordinate projections of $T$ into $\mathcal{H}$ and $\mathcal{K}$, respectively:

$$
P_{T}\{x, y\}:=x, \quad Q_{T}\{x, y\}:=y, \quad\{x, y\} \in T .
$$

Note that both $P_{T}$ and $Q_{T}$ are continuous (with norm bound 1) if we endow $T$ with the inner product coming from that of $\mathcal{H} \times \mathcal{K}$. We shall therefore call $P_{T}$ and $Q_{T}$ the canonical contractions of $T$. Assume in addition that $T$ is a closed relation, then the domain of $P_{T}$ and $Q_{T}$ becomes a Hilbert space and thus we may take the adjoint operators $P_{T}^{*}: \mathcal{H} \rightarrow T$ and $Q_{T}^{*}: \mathcal{K} \rightarrow T$, and also the product operators $P_{T} P_{T}^{*}, P_{T} Q_{T}^{*}, Q_{T} P_{T}^{*}$ and $Q_{T} Q_{T}^{*}$ are well defined contractions (Fig. 1).

The present paper is devoted to the study of these canonical contractions and their connection with the closed linear relation $T$. The paper is organized as follows. Section 2 contains a short overview of concepts that are needed in later sections of the paper. In Sect. 3 we analyse the first coordinate projection $P_{T}$. It turns out that the range of $P_{T}^{*}$ is identical with the restriction of $T_{\mathrm{s}}$, the so called regular part of $T$, to $\operatorname{dom} T^{*} T$. (We recall that the regular part $T_{\mathrm{s}}$ of $T$ is defined as the relation $T_{\mathrm{s}}:=\left(I-P_{m}\right) T$ where $P_{m}$ is the orthogonal projection of $\mathcal{K}$ onto mul $\bar{T}$, see [6].) Being so, the relation $\operatorname{ran} P_{T}^{*}$ is always the graph of a closable linear operator. In Sect. 4 we are going to establish some necessary and sufficient conditions for a pair $S, T$ of operators in order that they satisfy

$$
S^{*}=T \quad \text { and } \quad T^{*}=S,
$$

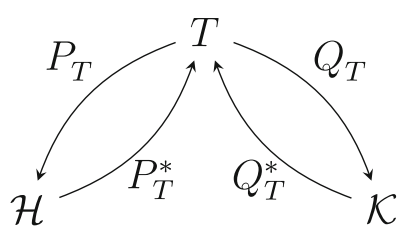

Fig. 1 The canonical graph contractions and their adjoints 
by means of the corresponding graph contractions $P_{T}, Q_{T}$ and $P_{S}, Q_{S}$, respectively. A different approach to the the same issue was treated in the recent paper [15] of the authors. The interested reader may also consult with $[10,11,13,14,16]$. In Sect. 5, we examine the appropriate products $P_{T} P_{T}^{*}, P_{T} Q_{T}^{*}, Q_{T} P_{T}^{*}$ and $Q_{T} Q_{T}^{*}$ of the contractions in question, and we are going to clarify their connection with the relations $T$ and $T^{*}$. In particular, we present a new and constructive proof of self-adjointness of $T^{*} T$ (provided that $T$ is closed) by showing that $\left(I+T^{*} T\right)^{-1}=P_{T} P_{T}^{*}$. Finally, we conclude the paper by showing how Stone's decomposition [17] of a closed linear relation $T$ can be obtain by applying the results.

\section{Linear Relations}

Throughout the paper, $\mathcal{H}$ and $\mathcal{K}$ will denote real or complex Hilbert spaces. A linear relation $T$ between $\mathcal{H}$ and $\mathcal{K}$ is nothing but a linear subspace of the product Hilbert space $\mathcal{H} \times \mathcal{K}$. We shall call the relation $T$ closed if it is a closed subspace of $\mathcal{H} \times \mathcal{K}$. Accordingly, the closure $\bar{T}$ of $T$ is always a closed linear relation, and being so, it becomes a Hilbert space with respect to the induced inner product

$$
(\{x, y\} \mid\{u, v\})_{T}:=(x \mid u)_{\mathcal{H}}+(y \mid v)_{\mathcal{K}}, \quad\{x, y\},\{u, v\} \in \bar{T} .
$$

If we refer to $\bar{T}$ as the above Hilbert space, we shall denote it by $\mathcal{G}(T)$.

Recall that every linear operator $T: \mathcal{H} \rightarrow \mathcal{K}$ when identified with its graph is a linear relation:

$$
T \equiv\{\{x, T x\}: x \in \operatorname{dom} T\} .
$$

Nevertheless, the closure (of the graph) of a linear operator is no longer an operator in general, namely, it may be that $\{0, k\} \in \bar{T}$ for some non-zero $k$. Accordingly, we call $T$ closable if its closure $\bar{T}$ is itself an operator.

The domain, range, kernel and multivalued part of a linear relation $T$ are defined to be the following linear subspaces, respectively:

$$
\begin{aligned}
\operatorname{dom} T: & =\{x \in \mathcal{H}:\{x, y\} \in T\}, & \operatorname{ran} T:=\{y \in \mathcal{K}:\{x, y\} \in T\}, \\
\operatorname{ker} T: & =\{x \in \mathcal{H}:\{x, 0\} \in T\}, & \operatorname{mul} T:=\{y \in \mathcal{K}:\{0, y\} \in T\} .
\end{aligned}
$$

It is immediate that $\operatorname{ker} T$ and mul $T$ are both closed subspaces whenever $T$ itself is closed. It goes also without saying that $T$ is (the graph of) an operator if and only if mul $T=\{0\}$, and that $T$ is (the graph of) a closable operator if and only of mul $\bar{T}=\{0\}$.

The inverse of a linear relation $T$ is defined as

$$
T^{-1}:=\{\{y, x\}:\{x, y\} \in T\} .
$$

If $S$ and $T$ are both linear relations then their product $T S$ is given by

$$
T S:=\{\{x, z\}:\{x, y\} \in S \text { and }\{y, z\} \in T \text { for some } y\} .
$$


The operatorlike sum of $S$ and $T$ is

$$
S+T:=\{\{x, y+z\}:\{x, y\} \in S,\{x, z\} \in T\},
$$

just like in the case of operators, while the componentwise (or Minkowski) sum is

$$
S \widehat{+} T:=\{\{x+v, y+z\}:\{x, y\} \in S,\{v, z\} \in T\} .
$$

The adjoint of a linear relation $T$ is defined by

$$
T^{*}:=W(T)^{\perp}
$$

where $W: \mathcal{H} \times \mathcal{K} \rightarrow \mathcal{K} \times \mathcal{H}$ is the 'flip' operator

$$
W\{h, k\}:=\{k,-h\}, \quad\{h, k\} \in \mathcal{H} \times \mathcal{K} .
$$

It is immediate that $T^{*}$ is a closed linear relation between $\mathcal{K}$ and $\mathcal{H}$ and that $T^{* *}=\bar{T}$. Note that the following orthogonal decomposition of $\mathcal{K} \times \mathcal{H}$ holds also true:

$$
T^{*} \widehat{\oplus} W\left(T^{* *}\right)=\mathcal{K} \times \mathcal{H}
$$

Another equivalent definition of $T^{*}$ might be given in terms of the inner product, namely,

$$
\{k, h\} \in T^{*} \Longleftrightarrow(y \mid k)_{\mathcal{K}}=(x \mid h)_{\mathcal{H}}, \quad \forall\{x, y\} \in T .
$$

Recall also the following identities:

$$
\operatorname{ker} T^{*}=(\operatorname{ran} T)^{\perp}, \quad \operatorname{mul} T^{*}=(\operatorname{dom} T)^{\perp} .
$$

For a given linear relation $T$, let us denote by $P_{m}$ the orthogonal projection of $\mathcal{K}$ onto mul $\bar{T}$. The regular part of $T$ is defined as the linear relation

$$
T_{\mathrm{s}}:=\left\{\left\{x,\left(I-P_{m}\right) y\right\}:\{x, y\} \in T\right\} .
$$

It can be shown that $T_{\mathrm{S}}$ is (the graph of) a closable operator. In contrast, the singular part

$$
T_{\text {sing }}:=\left\{\left\{x, P_{m} y\right\}:\{x, y\} \in T\right\}
$$

is a so called singular relation which means that $\overline{T_{\text {sing }}}$ is the product of two closed subspaces. By means of the regular and singular parts, the linear relation $T$ allows the following canonical sum decomposition

$$
T=T_{\mathrm{s}}+T_{\mathrm{sing}},
$$


see [6, Theorem 4.1]. Note also immediately that the regular and singular parts may be written as

$$
T_{\mathrm{s}}=\left(I-P_{m}\right) T, \quad T_{\text {sing }}=P_{m} T .
$$

We shall also use the fact that "regular part" and "closure" operations commute in the sense that

$$
\left(T_{\mathrm{s}}\right)^{* *}=\left(T^{* *}\right)_{\mathrm{s}}
$$

see [6, Proposition 4.5]. An important consequence of this result is that the regular part of a closed linear relation is closed itself, and also that $T_{\mathrm{S}} \subseteq T$, provided that $T$ is closed.

The interested reader is referred to the books [2,12] and papers $[1,6,7]$ where, in addition to the proofs of the above statements, more information about linear relations can be found.

\section{Canonical Graph Contractions of a Linear Relation}

Let $T$ be a linear relation between the real or complex Hilbert spaces $\mathcal{H}$ and $\mathcal{K}$. The canonical graph contractions $P_{T}: \bar{T} \rightarrow \mathcal{H}$ and $Q_{T}: \bar{T} \rightarrow \mathcal{K}$ of $T$ are defined as the mappings

$$
P_{T}\{x, y\}:=x, \quad Q_{T}\{x, y\}:=y, \quad\{x, y\} \in \bar{T} .
$$

Note that both of those mappings are linear contractions if we consider them as operators from the Hilbert space $\mathcal{G}(T)$ into $\mathcal{H}$ and $\mathcal{K}$, respectively:

$$
P_{T} \in \mathscr{B}(\mathcal{G}(T) ; \mathcal{H}),\left\|P_{T}\right\| \leq 1 \quad \text { and } \quad Q_{T} \in \mathscr{B}(\mathcal{G}(T) ; \mathcal{K}),\left\|Q_{T}\right\| \leq 1
$$

Therefore, their adjoint operators $P_{T}^{*} \in \mathscr{B}(\mathcal{H} ; \mathcal{G}(T))$ and $Q_{T}^{*} \in \mathscr{B}(\mathcal{K} ; \mathcal{G}(T))$ are themselves linear contractions, and their ranges $\operatorname{ran} P_{T}^{*}$ and $\operatorname{ran} Q_{T}^{*}$ are linear relations. Observe immediately that

$$
\operatorname{ker} P_{T}=\{0\} \times \operatorname{mul} \bar{T}, \quad \text { and } \quad \operatorname{ker} Q_{T}=\operatorname{ker} \bar{T} \times\{0\} .
$$

Consequently, we see that $T$ is (the graph) of a closable operator if and only if $P_{T}$ is one-to-one. The following identities

$$
\operatorname{ran} P_{T}=\operatorname{dom} \bar{T}, \quad \operatorname{ker} P_{T}^{*}=\operatorname{mul} T^{*}
$$

and

$$
\operatorname{ran} Q_{T}=\operatorname{ran} \bar{T}, \quad \operatorname{ker} Q_{T}^{*}=\operatorname{ker} T^{*}
$$


are also easy to verify. However, the descriptions of $\operatorname{ran} P_{T}^{*}$ and $\operatorname{ran} Q_{T}^{*}$ are somewhat more involved and need some preparations. We are going to deal with those subspaces in Theorem 3.6 and Proposition 5.5, respectively.

Note also that $P_{T}=P_{\bar{T}}$ and $Q_{T}=Q_{\bar{T}}$, by definition. Hence, there is no loss of generality in assuming that the linear relation $T$ is closed. In light of this, with a few exceptions, we will do so.

Below we are going to examine the properties of the contractions $P_{T}$ and $Q_{T}$ and their connection with $T$ in detail. We start out by analysing the first coordinate projection $P_{T}$.

Lemma 3.1 Let $T$ be a linear relation between $\mathcal{H}$ and $\mathcal{K}$, then

$$
\operatorname{ran} Q_{T} P_{T}^{*} \subseteq \operatorname{dom} T^{*}
$$

Proof Consider $h \in \mathcal{H}$ and let $P_{T}^{*} h:=\{z, w\} \in \operatorname{ran} P_{T}^{*}$, then we have

$$
(x \mid z)_{\mathcal{H}}+(y \mid w)_{\mathcal{K}}=\left(\{x, y\} \mid P_{T}^{*} h\right)_{T}=(x \mid h)_{\mathcal{H}},
$$

for every $\{x, y\} \in T$. Hence we get

$$
(y \mid w)_{\mathcal{K}}=(x \mid h-z)_{\mathcal{H}},
$$

which implies that $\{w, h-z\} \in T^{*}$ and therefore $w=Q_{T} P_{T}^{*} h \in \operatorname{dom} T^{*}$.

Proposition 3.2 Let $T$ be a linear relation between $\mathcal{H}$ and $\mathcal{K}$, then

$$
\operatorname{dom} T^{*} T=P_{T}\left(T \cap \operatorname{ran} P_{T}^{*}\right)
$$

Proof ' $\supseteq$ :' Assume first that $\{x, y\} \in T \cap \operatorname{ran} P_{T}^{*}$, then $y \in \operatorname{ran} Q_{T} P_{T}^{*} \subseteq \operatorname{dom} T^{*}$ by Lemma 3.1, and therefore there exists $z \in \mathcal{H}$ such that $\{y, z\} \in T^{*}$. This means that $\{x, z\} \in T^{*} T$ and therefore $x \in \operatorname{dom} T^{*} T$.

' $\subseteq$ :' Suppose on the converse that $x \in \operatorname{dom} T^{*} T$, then $\{x, y\} \in T$ and $\{y, z\} \in T^{*}$ for some $y$ and $z$. It suffices to show that $\{x, y\} \in \operatorname{ran} P_{T}^{*}$. Let therefore $\{u, v\} \in T$, then we have

$$
\begin{aligned}
(\{x, y\} \mid\{u, v\})_{T} & =(x \mid u)_{\mathcal{H}}+(y \mid v)_{\mathcal{K}}=(x \mid u)_{\mathcal{H}}+(z \mid u)_{\mathcal{H}} \\
& =\left(x+z \mid P_{T}\{u, v\}\right)_{\mathcal{H}}=\left(P_{T}^{*}(x+z) \mid\{u, v\}\right)_{T},
\end{aligned}
$$

whence it follows that $\{x, y\}=P_{T}^{*}(x+z) \in \operatorname{ran} P_{T}^{*}$.

From the above proposition we get the following two straightforward corollaries:

Corollary 3.3 If $T$ is (the graph of) an operator, then

$$
\left.T\right|_{\operatorname{dom} T^{*} T}=T \cap \operatorname{ran} P_{T}^{*} .
$$

If $T$ is closed in addition, then

$$
\left.T\right|_{\text {dom } T^{*} T}=\operatorname{ran} P_{T}^{*} .
$$


Corollary 3.4 If $T$ is a closed linear relation then

$$
\operatorname{dom} T^{*} T=\operatorname{dom}\left(\operatorname{ran} P_{T}^{*}\right)
$$

At the beginning of this section we determined the subspaces ker $P_{T}, \operatorname{ran} P_{T}$ and ker $P_{T}^{*}$ [see formulas (3.1) and (3.2)]. Next we deal with the range space of $P_{T}^{*}$. Observe that ran $P_{T}^{*}$, being a linear subspace of $\bar{T}$, is itself a linear relation. In the ensuing result we shall see that ran $P_{T}^{*}$ is, in fact, (the graph of) a closable operator.

Theorem 3.5 Let $T$ be a linear relation between $\mathcal{H}$ and $\mathcal{K}$, then

$$
\overline{\operatorname{ran} P_{T}^{*}}=\overline{T_{\mathrm{s}}} .
$$

In particular, ran $P_{T}^{*}$ is always (the graph of) a closable operator.

Proof First of all note that if $\left\{x,\left(I-P_{m}\right) y\right\} \in \bar{T}$ for some $\{x, y\} \in \mathcal{H} \times \mathcal{K}$, then necessarily $\{x, y\} \in \bar{T}$. (Recall that $P_{m}$ stands for the orthogonal projection of $\mathcal{K}$ onto mul $\bar{T}$.) Indeed,

$$
\{x, y\}=\left\{x,\left(I-P_{m}\right) y\right\}+\left\{0, P_{m} y\right\} \in \bar{T} \widehat{+}(\{0\} \times \operatorname{mul}(\bar{T})) \subseteq \bar{T}+\bar{T}=\bar{T} .
$$

Recall that by (3.1) we have ker $P_{T}=\{0\} \times \operatorname{mul} \bar{T}$. Hence we have

$$
\begin{aligned}
\overline{\operatorname{ran} P_{T}^{*}} & =(\{0\} \times \operatorname{mul} \bar{T})^{\perp} \\
& =\left\{\{x, y\} \in \bar{T}: y \in(\operatorname{mul} \bar{T})^{\perp}\right\} \\
& =\left\{\{x, y\} \in \bar{T}: y \in \operatorname{ran}\left(I-P_{m}\right)\right\} \\
& =\left\{\left\{x,\left(I-P_{m}\right) v\right\}: v \in \mathcal{K},\left\{x,\left(I-P_{m}\right) v\right\} \in \bar{T}\right\} \\
& =\left\{\left\{x,\left(I-P_{m}\right) v\right\}:\{x, v\} \in \bar{T}\right\} \\
& =(\bar{T})_{\mathrm{s}}=\overline{T_{\mathrm{s}}},
\end{aligned}
$$

as it is claimed.

Theorem 3.6 For every linear relation $T$ between $\mathcal{H}$ and $\mathcal{K}$ we have

$$
\operatorname{ran} P_{T}^{*}=\left.\left(\overline{T_{\mathrm{s}}}\right)\right|_{\operatorname{dom} T^{*} \bar{T}} .
$$

Proof Note that we have identity $P_{T}=P_{\bar{T}}$ for every linear relation. On the other hand, $\overline{T_{\mathrm{s}}}=(\bar{T})_{\mathrm{s}}$ according to (2.6). Therefore, without loss of generality we may assume that $T$ is closed, in which case (3.4) reduces to

$$
\operatorname{ran} P_{T}^{*}=\left.\left(T_{\mathrm{s}}\right)\right|_{\operatorname{dom} T^{*} T}
$$

By Theorem 3.5 we have $\operatorname{ran} P_{T}^{*} \subseteq T_{\mathrm{S}}$ and by Corollary 3.4, $\operatorname{dom}\left(\operatorname{ran} P_{T}^{*}\right)=\operatorname{dom} T^{*} T$ whenever $T$ is closed. Since $T_{\mathrm{s}}$ is the graph of an operator, we obtain (3.5). 


\section{Linear Relations Adjoint to Each Other}

Let $T$ and $S$ be linear relations between $\mathcal{H}$ and $\mathcal{K}$, respectively, $\mathcal{K}$ and $\mathcal{H}$. We say that $T$ and $S$ are adjoint to each other (or that $T, S$ form an adjoint pair), if they satisfy

$$
T \subset S^{*} \quad \text { and } \quad S \subset T^{*}
$$

or equivalently, if

$$
(y \mid v)_{\mathcal{K}}=(x \mid u)_{\mathcal{H}}, \quad \forall\{x, y\} \in T, \quad \forall\{v, u\} \in S
$$

An important and natural question is under what conditions are the equations $T=S^{*}$ and $S=T^{*}$ satisfied. Below we provide some necessary and sufficient conditions on the pair $S, T$ by means of the corresponding graph contractions $P_{T}, Q_{T}$ and $P_{S}, Q_{S}$ in order that they satisfy the weaker identities $T^{* *}=S^{*}$ and $S^{* *}=T^{*}$

Theorem 4.1 Let $S, T$ be linear relations between $\mathcal{H}$ and $\mathcal{K}$, respectively, $\mathcal{K}$ and $\mathcal{H}$, that are adjoint to each other in the sense of (4.1). Then the following statements are equivalent:

(i) $S^{*}=T^{* *}$ and $T^{*}=S^{* *}$,

(ii) (a) $P_{T} P_{T}^{*}+Q_{S} Q_{S}^{*}=I_{\mathcal{H}}$,

(b) $P_{S} P_{S}^{*}+Q_{T} Q_{T}^{*}=I_{\mathcal{K}}$,

(c) $Q_{T} P_{T}^{*}=P_{S} Q_{S}^{*}$.

Proof Since the corresponding canonical contractions of $T$ and $\bar{T}$ (resp., of $S$ and $\bar{S}$ ) are identical, we may assume without loss of generality that both $S$ and $T$ are closed. Let us introduce the following operator matrix

$$
U_{T, S}:=\left[\begin{array}{cc}
P_{T} & -Q_{S} \\
Q_{T} & P_{S}
\end{array}\right]: \mathcal{G}(T) \times \mathcal{G}(S) \rightarrow \mathcal{H} \times \mathcal{K},
$$

which acts between $\bar{T} \times \bar{S}$ and $\mathcal{H} \times \mathcal{K}$ by the correspondence

$$
U_{T, S}\left[\begin{array}{l}
\{x, y\} \\
\{v, u\}
\end{array}\right]:=\{x-u, y+v\}, \quad\{x, y\} \in T,\{v, u\} \in S .
$$

Since $T$ and $S$ are adjoint to each other, one concludes that $U_{T, S}$ is an isometry: for let $\{x, y\} \in T$ and $\{v, u\} \in S$, then by (4.2)

$$
\begin{aligned}
\left\|U_{T, S}\left[\begin{array}{l}
\{x, y\} \\
\{v, u\}
\end{array}\right]\right\|^{2} & =\|x-u\|^{2}+\|y+v\|^{2} \\
& =\|x\|^{2}+\|y\|^{2}+\|v\|^{2}+\|u\|^{2}+2 \operatorname{Re}\left[(y \mid v)_{\mathcal{K}}-(x \mid u)_{\mathcal{H}}\right] \\
& =\left\|\left[\begin{array}{l}
\{x, y\} \\
\{v, u\}
\end{array}\right]\right\|^{2} .
\end{aligned}
$$


Observe immediately that the range of $U_{T, S}$ can be written as

$$
\operatorname{ran} U_{T, S}=T \widehat{\oplus} W(S),
$$

where $W$ is the 'flip' operator (2.1) and $\widehat{\oplus}$ denotes orthogonal Minkowski sum. On the other hand, we have the orthogonal decomposition

$$
\mathcal{H} \times \mathcal{K}=T \widehat{\oplus} W\left(T^{*}\right)
$$

for the closed linear relation $T$. From these last two identities it follows that $U_{T, S}$ is unitary if and only if $W(S)=W\left(T^{*}\right)$, i.e., $S=T^{*}$. An easy calculation shows that

$$
U_{T, S} U_{T, S}^{*}=\left[\begin{array}{ll}
P_{T} P_{T}^{*}+Q_{S} Q_{S}^{*} & P_{T} Q_{T}^{*}-Q_{S} P_{S}^{*} \\
Q_{T} P_{T}^{*}-P_{S} Q_{S}^{*} & P_{S} P_{S}^{*}+Q_{T} Q_{T}^{*}
\end{array}\right]
$$

from which the desired equivalence between (i) and (ii) already follows.

Remark 4.2 Some characterizations of those linear operators $S, T$ which satisfy identities $S^{*}=T$ and $T^{*}=S$ were given in [15] by means of the operator matrix

$$
M_{T, S}:=\left[\begin{array}{cc}
I_{\mathcal{H}} & -S \\
T & I_{\mathcal{K}}
\end{array}\right]
$$

cf. also [10,14]. The general case of linear relations was discussed in [11] in the same spirit. For an exact interpretation of matrices with linear relation entries the reader is referred to [8].

\section{Products of Graph Contractions}

Let $T$ be linear relation between the Hilbert spaces $\mathcal{H}$ and $\mathcal{K}$ and consider its canonical graph contractions $P_{T}: \mathcal{G}(T) \rightarrow \mathcal{H}$ and $Q_{T}: \mathcal{G}(T) \rightarrow \mathcal{K}$. Then the following four operators $P_{T} P_{T}^{*}, P_{T} Q_{T}^{*}, Q_{T} P_{T}^{*}$ and $Q_{T} Q_{T}^{*}$ are all well defined linear contractions between the appropriate Hilbert spaces. In this section we clarify their role and connection with the relations $T$ and $T^{*}$.

Lemma 5.1 Let $T$ be a closed linear relation between $\mathcal{H}$ and $\mathcal{K}$, then

(a) $\operatorname{mul}\left(T T^{*}\right)=\operatorname{mul} T$,

(b) $T^{*} T=\left(T_{\mathrm{s}}\right)^{*} T_{\mathrm{s}}$,

(c) $\left(T T^{*}\right)_{\mathrm{s}}=T_{\mathrm{s}}\left(T^{*}\right)_{\mathrm{s}}$.

Proof (a) Let $k \in \operatorname{mul} T$, then $\{0,0\} \in T^{*}$ and $\{0, k\} \in T$ implies that $k \in \operatorname{mul} T T^{*}$. Assume on the converse that $k \in \operatorname{mul} T T^{*}$, then there exists $u$ such that $\{0, u\} \in T^{*}$ and $\{u, k\} \in T$. Since we have $u \in \operatorname{dom} T \cap \operatorname{mul} T^{*}$, from (2.3) it follows that $u=0$. So $\{0, k\} \in T$ and therefore $k \in \operatorname{mul} T$. 
(b) First we show inclusion $T^{*} T \subset\left(T_{\mathrm{S}}\right)^{*} T_{\mathrm{s}}$. Take $\{x, z\} \in T^{*} T$, then there exists $y$ such that $\{x, y\} \in T$ and $\{y, z\} \in T^{*}$. In particular we have $y \in \operatorname{dom} T^{*} \subseteq$ $\operatorname{mul} T^{\perp}$, thus $\{x, y\}=\left\{x,\left(I-P_{m}\right) y\right\} \in T_{\mathrm{S}}$. On the other hand, we have inclusion $T_{\mathrm{S}} \subset T$ by closedness, so $T^{*} \subset\left(T_{\mathrm{S}}\right)^{*}$. Consequently, $\{x, z\} \in\left(T_{\mathrm{S}}\right)^{*} T_{\mathrm{S}}$, indeed. Conversely, let $\{x, z\} \in\left(T_{\mathrm{S}}\right)^{*} T_{\mathrm{S}}$, then there exists $y$ such that $\{x, y\} \in T_{\mathrm{S}}$ and $\{y, z\} \in\left(T_{\mathrm{S}}\right)^{*}$. Here we have $\{x, y\} \in T$ as $T_{\mathrm{s}} \subseteq T$. Furthermore, $\left(T_{\mathrm{s}}\right)^{*}$ can be written as

$$
\left(T_{\mathrm{s}}\right)^{*}=T^{*} \widehat{\oplus}(\operatorname{mul} T \times\{0\}),
$$

where $\widehat{\oplus}$ denotes Minkowski direct sum. This yields us $\{k, h\} \in T^{*}$ and $w \in \operatorname{mul} T$ such that $\{y, z\}=\{k, h\}+\{w, 0\}$. Since $y, k \in \operatorname{mul} T^{\perp}$, we get $w=0$ and $y=k$, consequently $\{y, z\} \in T^{*}$ and $\{x, z\} \in T^{*} T$.

(c) By (a) we have mul $T=\operatorname{mul}\left(T T^{*}\right)$, hence

$$
\left(T T^{*}\right)_{\mathrm{s}}=\left(I-P_{m}\right) T T^{*}=T_{\mathrm{s}} T^{*} \supset T_{\mathrm{s}}\left(T^{*}\right)_{\mathrm{s}}
$$

because $\left(T^{*}\right)_{\mathrm{s}} \subset T^{*}$. To see the converse inclusion take $\left\{v,\left(I-P_{m}\right) w\right\} \in\left(T T^{*}\right)_{\mathrm{s}}$, and let $\{v, u\} \in T^{*}$ and $\{u, w\} \in T$ for some $u$, then $\left\{u,\left(I-P_{m}\right) w\right\} \in T_{\mathrm{S}}$ and from $u \in \operatorname{dom} T$ we get that $u \in(\operatorname{mul} \bar{T})^{\perp}$, hence $\{v, u\} \in\left(T^{*}\right)_{\mathrm{s}}$. Thus $\left\{v,\left(I-P_{m}\right) w\right\} \in T_{\mathrm{s}}\left(T^{*}\right)_{\mathrm{s}}$.

In the next theorem we are going to deal with the contractions $P_{T} P_{T}^{*}, Q_{T} Q_{T}^{*}, P_{T} Q_{T}^{*}$ and $Q_{T} P_{T}^{*}$.

Theorem 5.2 Let $T$ be a closed linear relation between $\mathcal{H}$ and $\mathcal{K}$. Then

(a) $P_{T} P_{T}^{*}=\left(I+T^{*} T\right)^{-1}$,

(b) $Q_{T} P_{T}^{*}=T_{\mathrm{s}}\left(I+T^{*} T\right)^{-1}$,

(c) $P_{T} Q_{T}^{*}=\left(T^{*}\right)_{\mathrm{S}}\left(I+T T^{*}\right)^{-1}$,

(d) $Q_{T} Q_{T}^{*}=I-\left(I+T T^{*}\right)^{-1}=P_{m}+\left(T T^{*}\right)_{\mathrm{s}}\left(I+T T^{*}\right)^{-1}$.

Proof (a) Let us introduce the linear operator

$$
P_{T}^{\dagger}: \operatorname{dom} T \rightarrow \mathcal{H} \times \mathcal{K}, \quad P_{T}^{\dagger} u:=\left\{u, T_{\mathrm{s}} u\right\}
$$

Observe that $P_{T}^{\dagger} u \in T_{\mathrm{s}} \subseteq T$ for every $u \in \operatorname{dom} T$, and that

$$
P_{T}^{\dagger} P_{T}\left\{u, T_{\mathrm{s}} u\right\}=\left\{u, T_{\mathrm{s}} u\right\}
$$

Since ran $P_{T}^{*} \subset T_{\mathrm{s}}$ by Theorem 3.5, from (5.1) it follows that $P_{T}^{\dagger} P_{T} P_{T}^{*}=P_{T}^{*}$. Let now $x \in \operatorname{dom} T$ and $h \in \mathcal{H}$, then

$$
\begin{aligned}
(x \mid h)_{\mathcal{H}} & =\left(P_{T} P_{T}^{\dagger} x \mid h\right)_{\mathcal{H}}=\left(P_{T}^{\dagger} x \mid P_{T}^{*} h\right)_{T}=\left(P_{T}^{\dagger} x \mid P_{T}^{\dagger} P_{T} P_{T}^{*} h\right)_{T} \\
& =\left(\left\{x, T_{\mathrm{s}} x\right\} \mid\left\{P_{T} P_{T}^{*} h, T_{\mathrm{s}} P_{T} P_{T}^{*} h\right\}\right)_{T} \\
& =\left(x \mid P_{T} P_{T}^{*} h\right)_{\mathcal{H}}+\left(T_{\mathrm{s}} x \mid T_{\mathrm{s}} P_{T} P_{T}^{*} h\right)_{\mathcal{K}},
\end{aligned}
$$


consequently,

$$
\left(T_{\mathrm{s}} x \mid T_{\mathrm{s}} P_{T} P_{T}^{*} h\right)_{\mathcal{K}}=\left(x \mid h-P_{T} P_{T}^{*} h\right)_{\mathcal{H}} \cdot
$$

This implies that

$$
P_{T} P_{T}^{*} h \in \operatorname{dom}\left(T_{\mathrm{s}}\right)^{*} T_{\mathrm{s}} \quad \text { and } \quad h=\left(I+\left(T_{\mathrm{s}}\right)^{*} T_{\mathrm{s}}\right) P_{T} P_{T}^{*} h,
$$

that is, $P_{T} P_{T}^{*}=\left(I+\left(T^{*}\right)_{\mathrm{s}} T_{\mathrm{s}}\right)^{-1}$. Since we have identity $T^{*} T=\left(T^{*}\right)_{\mathrm{s}} T_{\mathrm{s}}$ by Lemma 5.1, the proof of part (a) is complete.

(b) Take any vector $h \in \mathcal{H}$. From (a) and equality $P_{T}^{\dagger} P_{T} P_{T}^{*}=P_{T}^{*}$ we conclude that

$$
\begin{aligned}
Q_{T} P_{T}^{*} h & =Q_{T} P_{T}^{\dagger} P_{T} P_{T}^{*} h=Q_{T} P_{T}^{\dagger}\left(I+T^{*} T\right)^{-1} h \\
& =Q_{T}\left\{\left(I+T^{*} T\right)^{-1} h, T_{\mathrm{S}}\left(I+T^{*} T\right)^{-1} h\right\} \\
& =T_{\mathrm{S}}\left(I+T^{*} T\right)^{-1} h,
\end{aligned}
$$

whence we get identity (b).

(c) Replacing $T$ by $T^{*}$ in (b), we obtain that

$$
Q_{T^{*}} P_{T^{*}}^{*}=\left(T^{*}\right)_{\mathrm{s}}\left(I+T T^{*}\right)^{-1} .
$$

On the other hand, it follows from Theorem 4.1 (ii) (c) that $P_{T} Q_{T}^{*}=Q_{T^{*}} P_{T^{*}}^{*}$, hence the desired identity follows.

(d) First we note that

$$
\left(I-P_{m}\right) Q_{T}=T_{\mathrm{S}} P_{T},
$$

because for $\{x, y\} \in T$,

$$
\left(I-P_{m}\right) Q_{T}\{x, y\}=\left(I-P_{m}\right) y=T_{\mathrm{s}} x=T_{\mathrm{s}} P_{T}\{x, y\} .
$$

From this and (c) we get that

$$
\begin{aligned}
\left(I-P_{m}\right) Q_{T} Q_{T}^{*} & =T_{\mathrm{s}} P_{T} Q_{T}^{*}=T_{\mathrm{s}}\left(T^{*}\right)_{\mathrm{S}}\left(I+T T^{*}\right)^{-1} \\
& =\left(T T^{*}\right)_{\mathrm{S}}\left(I+T T^{*}\right)^{-1}
\end{aligned}
$$

On the other hand, we have

$$
Q_{T} Q_{T}^{*}=I-P_{T^{*}} P_{T^{*}}^{*},
$$

by Theorem 4.1 (ii) (b). Since ran $P_{T^{*}}=\operatorname{dom} T^{*} \subset(\operatorname{mul} T)^{\perp}$, we get

$$
P_{m} Q_{T} Q_{T}^{*}=P_{m}-P_{m} P_{T^{*}} P_{T^{*}}^{*}=P_{m} .
$$


From the above identities we get

$$
Q_{T} Q_{T}^{*}=P_{m} Q_{T} Q_{T}^{*}+\left(I-P_{m}\right) Q_{T} Q_{T}^{*}=P_{m}+\left(T T^{*}\right)_{\mathrm{S}}\left(I+T T^{*}\right)^{-1},
$$

which completes the proof.

Remark 5.3 We notice that $P_{T}^{\dagger}$ appearing in the proof of the preceding theorem is identical with the Moore-Penrose inverse of $P_{T}$, cf. [3] or [4]. We also remark that the proof might be slightly simplified when $T$ is a closed operator. Namely, in that case we have $T=T_{\mathrm{s}}$ and $P_{T}^{\dagger}=P_{T}^{-1}$.

Corollary 5.4 Let $T$ be a densely defined closed linear operator between two Hilbert spaces. Then

(a) $P_{T} P_{T}^{*}=\left(I+T^{*} T\right)^{-1}$,

(b) $Q_{T} P_{T}^{*}=T\left(I+T^{*} T\right)^{-1}$,

(c) $P_{T} Q_{T}^{*}=T^{*}\left(I+T T^{*}\right)^{-1}$,

(d) $Q_{T} Q_{T}^{*}=T T^{*}\left(I+T T^{*}\right)^{-1}$.

Proof The proof is straightforward from Theorem 5.2 by noticing that $P_{m}=0$ whenever $T$ is a densely defined closed operator.

At the beginning of Sect. 3 we determined the subspaces $\operatorname{ker} Q_{T}, \operatorname{ker} Q_{t}^{*}$ and ran $Q_{T}$. Now, applying Theorem 5.2 we are able to give a description of the range space $\operatorname{ran} Q_{T}^{*}$ as well:

Proposition 5.5 For every linear relation $T$ between $\mathcal{H}$ and $\mathcal{K}$ we have

$$
\operatorname{ran} Q_{T}^{*}=\left.\left(\overline{T_{\mathrm{s}}}\right)\right|_{\operatorname{ran} T^{*} \cap \operatorname{dom} \bar{T}} \widehat{\oplus}(\{0\} \times \operatorname{mul} \bar{T})
$$

Proof Throughout the proof we may and will assume that $T$ is closed. According to Theorem 5.2 (c) and (d) we have

$$
\begin{aligned}
\operatorname{ran} Q_{T}^{*} & =\left\{\left\{P_{T} Q_{T}^{*} k, Q_{T} Q_{T}^{*} k\right\}: k \in \mathcal{K}\right\} \\
& =\left\{\left\{\left(T^{*}\right)_{\mathrm{S}}\left(I+T T^{*}\right)^{-1} k, T_{\mathrm{S}}\left(T^{*}\right)_{\mathrm{S}}\left(I+T T^{*}\right)^{-1} k+P_{m} k: k \in \mathcal{K}\right\}\right. \\
& =\left\{\left\{\left(T^{*}\right)_{\mathrm{S}}\left(I+T T^{*}\right)^{-1} k,\left(T T^{*}\right)_{\mathrm{S}}\left(I+T T^{*}\right)^{-1} k\right\}: k \in \mathcal{K}\right\} \widehat{+}(\{0\} \times \operatorname{mul} T),
\end{aligned}
$$

where in the last equality we used that

$$
\operatorname{ker}\left(I+T T^{*}\right)^{-1}=\operatorname{mul} T T^{*}=\operatorname{mul} T=\operatorname{ran} P_{m} .
$$

We have on the other hand

$$
\begin{aligned}
& \left\{\left\{\left(T^{*}\right)_{\mathrm{S}}\left(I+T T^{*}\right)^{-1} k,\left(T T^{*}\right)_{\mathrm{S}}\left(I+T T^{*}\right)^{-1} k\right\}: k \in \mathcal{K}\right\} \\
& \quad=\left\{\left\{\left(T^{*}\right)_{\mathrm{S}} z, T_{\mathrm{S}}\left(T^{*}\right)_{\mathrm{s}} z\right\}: z \in \operatorname{dom} T_{\mathrm{S}}\left(T^{*}\right)_{\mathrm{s}}\right\} \\
& \quad=\left.T_{\mathrm{S}}\right|_{\operatorname{ran}\left(T^{*}\right)_{\mathrm{S}} \cap \operatorname{dom} T_{\mathrm{S}} .}
\end{aligned}
$$


Finally we note that $\operatorname{dom} T_{\mathrm{s}}=\operatorname{dom} T$ and that $\operatorname{mul} T^{*}=\overline{\operatorname{dom} T}$ because $T$ is closed. Therefore

$$
\operatorname{ran}\left(T^{*}\right)_{\mathrm{S}} \cap \operatorname{dom} T_{\mathrm{s}}=\operatorname{ran} T^{*} \cap \operatorname{dom} T .
$$

This together with the above observations yields identity (5.2).

Remark 5.6 One of the anonymous referees suggested the following alternative proof of Proposition 5.5. Observe that we have

$$
Q_{T}=P_{T^{-1}} V,
$$

where $V: T \rightarrow T^{-1}$ is the unitary operator $V\{x, y\}:=\{y, x\}$. Hence

$$
\operatorname{ran} Q_{T}^{*}=\operatorname{ran} V^{-1} P_{T^{-1}}^{*}
$$

Using Theorem 3.6 we have

$$
\operatorname{ran} P_{T^{-1}}^{*}=\left.\left(T^{-1}\right)_{\mathrm{S}}\right|_{\mathrm{dom}\left(T^{-1}\right)^{*} T^{-1}}
$$

where the regular part of $T^{-1}$ can be written in the form

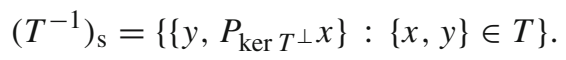

Using this and (5.3), with some calculation formula (5.2) can be deduced.

Corollary 5.7 Let $T$ be a closed linear relation between $\mathcal{H}$ and $\mathcal{K}$, then

$$
T_{\mathrm{S}}=\left.\left.T_{\mathrm{S}}\right|_{\operatorname{dom} T^{*} T} \widehat{+} T_{\mathrm{s}}\right|_{\operatorname{ran} T^{*} \cap \operatorname{dom} T} .
$$

If $T$ is a closed operator, then

$$
T=\left.\left.T\right|_{\operatorname{dom} T^{*} T} \widehat{+} T\right|_{\operatorname{ran} T^{*} \cap \operatorname{dom} T} .
$$

Proof Recall that the operator matrix $U_{T, T^{*}}$ defined by (4.3) with $S:=T^{*}$ is an isometry hence, in particular one has $P_{T}^{*} P_{T}+Q_{T}^{*} Q_{T}=I_{\mathcal{G}(T)}$. As a consequence, we have by [5, Theorem 2.2] that

$$
\begin{aligned}
T & =\operatorname{ran}\left(P_{T}^{*} P_{T}+Q_{T}^{*} Q_{T}\right)^{1 / 2}=\operatorname{ran} P_{T}^{*} \widehat{+} \operatorname{ran} Q_{T}^{*} \\
& =\left.\left.T_{\mathrm{S}}\right|_{\operatorname{dom} T^{*} T} \widehat{+} T_{\mathrm{s}}\right|_{\operatorname{ran} T^{*} \cap \operatorname{dom} T} \widehat{+}(\{0\} \times \operatorname{mul} T) .
\end{aligned}
$$

Now the desired identity follows since $T_{\mathrm{S}}=T \widehat{\ominus}(\{0\} \times \operatorname{mul} T)$. 
We conclude the paper with an application of the results. Let $T$ be a closed linear relation between the Hilbert spaces $\mathcal{H}$ and $\mathcal{K}$ and denote by $E_{T}$ the orthogonal projection of $\mathcal{H} \times \mathcal{K}$ onto $T$. Then $E_{T}$ admits a matrix representation as an operator in $\mathcal{H} \times \mathcal{K}$ :

$$
E_{T}=\left[\begin{array}{ll}
E_{11} & E_{12} \\
E_{21} & E_{22}
\end{array}\right]
$$

where the components $E_{i j}$ are bounded operators between the appropriate Hilbert spaces. Recall that $E_{T}$ is called the characteristic projection of $T$ by Stone, who proved that the entries $E_{i j}$ may be expressed in terms of $T$ and $T^{*}$, provided that $T$ is a densely defined and closed operator (see [17, Theorem 4], cf. also [9, Theorem 3]):

$$
E_{T}=\left[\begin{array}{cc}
\left(T^{*} T+I\right)^{-1} & T^{*}\left(T T^{*}+I\right)^{-1} \\
T\left(T^{*} T+I\right)^{-1} & T T^{*}\left(T T^{*}+I\right)^{-1}
\end{array}\right]
$$

In [6, Lemma 6.4], the above result of Stone was extended to closed linear relations. In the ensuing theorem we are going to restate this general result as a straightforward consequence of Theorem 5.2:

Theorem 5.8 Let $T$ be a closed linear relation between the Hilbert spaces $\mathcal{H}$ and $\mathcal{K}$. Then the characteristic projection $E_{T}$ of $T$ can be written as

$$
E_{T}=\left[\begin{array}{cc}
P_{T} P_{T}^{*} & P_{T} Q_{T}^{*} \\
Q_{T} P_{T}^{*} & Q_{T} Q_{T}^{*}
\end{array}\right]=\left[\begin{array}{cc}
\left(T^{*} T+I\right)^{-1} & \left(T^{*}\right)_{\mathrm{S}}\left(T T^{*}+I\right)^{-1} \\
T_{\mathrm{S}}\left(T^{*} T+I\right)^{-1} & I-\left(T T^{*}+I\right)^{-1}
\end{array}\right]
$$

Proof Consider the canonical embedding operator $V_{T}: \mathcal{G}(T) \rightarrow \mathcal{H} \times \mathcal{K}$, given by

$$
V_{T}:=\left[\begin{array}{c}
P_{T} \\
Q_{T}
\end{array}\right]\{x, y\}:=\{x, y\}, \quad\{x, y\} \in T .
$$

Clearly, $V_{T}$ is a linear isometry with range $T$ and therefore $V_{T} V_{T}^{*}$ is identical with $E_{T}$, i.e.,

$$
E_{T}=\left[\begin{array}{c}
P_{T} \\
Q_{T}
\end{array}\right]\left[\begin{array}{ll}
P_{T}^{*} & Q_{T}^{*}
\end{array}\right]=\left[\begin{array}{cc}
P_{T} P_{T}^{*} & P_{T} Q_{T}^{*} \\
Q_{T} P_{T}^{*} & Q_{T} Q_{T}^{*}
\end{array}\right]
$$

Theorem 5.2 completes now the proof.

Acknowledgements The authors are extremely grateful to the anonymous referees for reading the paper carefully and for their enlightening comments which improved the exposition of the manuscript.

Funding Open access funding provided by Eötvös Loránd University.

Data Availability Data sharing not applicable to this article as no datasets were generated or analysed during the current study. 
Open Access This article is licensed under a Creative Commons Attribution 4.0 International License, which permits use, sharing, adaptation, distribution and reproduction in any medium or format, as long as you give appropriate credit to the original author(s) and the source, provide a link to the Creative Commons licence, and indicate if changes were made. The images or other third party material in this article are included in the article's Creative Commons licence, unless indicated otherwise in a credit line to the material. If material is not included in the article's Creative Commons licence and your intended use is not permitted by statutory regulation or exceeds the permitted use, you will need to obtain permission directly from the copyright holder. To view a copy of this licence, visit http://creativecommons.org/licenses/by/4.0/.

\section{References}

1. Arens, R.: Operational calculus of linear relations. Pac. J. Math. 11, 9-23 (1961)

2. Behrndt, J., Hassi, S., de Snoo, H.S.V.: Boundary value problems, Weyl functions, and differential operators. Springer Nature, Monographs in Mathematics, p. 772 (2020)

3. Ben-Israel, A., Greville, T.N.E.: Generalized Inverses: Theory and Applications, vol. 15. Springer, London (2003)

4. Beutler, F.J.: Operator theory of the pseudo-inverse II. Unbounded operators with arbitrary range. J. Math. Anal. Appl. 10, 471-493 (1965)

5. Fillmore, P.A., Williams, J.P.: On operator ranges. Adv. Math. 7, 254-281 (1971)

6. Hassi, S., Sebestyén, Z., de Snoo, H.S.V., Szafraniec, F.H.: A canonical decomposition for linear operators and linear relations. Acta Math. Hungar. 115, 281-307 (2007)

7. Hassi, S., de Snoo, H.S.V., Szafraniec, F.H.: Componentwise and canonical decompositions of linear relations. Dissertationes Math. 465, 59 (2009)

8. Hassi, S., Labrousse, J-Ph, de Snoo, H.S.V.: Operational calculus for rows, columns, and blocks of linear relations. Adv. Oper. Theory 5, 1193-1228 (2020)

9. Jorgensen, P.E.T., Pearse, E., Tian, F.: Unbounded operators in Hilbert space, duality rules, characteristic projections, and their applications. Anal. Math. Phys. 8, 351-382 (2018)

10. Popovici, D., Sebestyén, Z.: Operators which are adjoint to each other. Acta Sci. Math. (Szeged) 80, 175-194 (2014)

11. Sandovici, A.: On the Adjoint of linear relations in Hilbert spaces. Mediterr. J. Math. 17, 68 (2020)

12. Schmüdgen, K.: Unbounded Self-adjoint Operators on Hilbert Space, vol. 265. Springer, London (2012)

13. Sebestyén, Z., Tarcsay, Z.: Characterizations of selfadjoint operators. Stud. Sci. Math. Hungar. 50, 423-435 (2013)

14. Sebestyén, Z., Tarcsay, Z.: Adjoint of sums and products of operators in Hilbert spaces. Acta Sci. Math. (Szeged) 82, 175-191 (2016)

15. Sebestyén, Z., Tarcsay, Z.: On the adjoint of Hilbert space operators. Linear Multilinear Algebra 67, 625-645 (2019)

16. Sebestyén, Z., Tarcsay, Z.: Range-kernel characterizations of operators which are adjoint of each other. Adv. Oper. Theory 5, 1026-1038 (2020)

17. Stone, M.H.: On unbounded operators in Hilbert spaces. J. Indian Math. Soc. 15, 155-192 (1952)

Publisher's Note Springer Nature remains neutral with regard to jurisdictional claims in published maps and institutional affiliations. 


\section{Affiliations}

\section{Zsigmond Tarcsay $^{1,2}\left[\right.$ [D $\cdot$ Zoltán Sebestyén $^{1}$}

Zoltán Sebestyén sebesty@cs.elte.hu

1 Department of Applied Analysis and Computational Mathematics, Eötvös Loránd University, Pázmány Péter sétány 1/c, Budapest 1117, Hungary

2 Alfréd Rényi Institute of Mathematics, Reáltanoda utca 13-15, Budapest 1053, Hungary 